\title{
Familial Retinoblastoma: Raised Awareness Improves Early Diagnosis and Outcome
}

\author{
Ibrahim Al-Nawaiseh, Aseel Q. Ghanem, and Yacoub A. Yousef \\ Department of Surgery, King Hussein Cancer Center, Amman, Jordan \\ Correspondence should be addressed to Yacoub A. Yousef; drjaqub@yahoo.com
}

Received 8 September 2016; Revised 29 November 2016; Accepted 22 January 2017; Published 1 March 2017

Academic Editor: Lawrence S. Morse

Copyright (C) 2017 Ibrahim Al-Nawaiseh et al. This is an open access article distributed under the Creative Commons Attribution License, which permits unrestricted use, distribution, and reproduction in any medium, provided the original work is properly cited.

Purpose. To study the impact of awareness of retinoblastoma in the affected families on the management and outcome of familial retinoblastoma patients. Methods and Materials. This is a retrospective, clinical case series of 44 patients with familial retinoblastoma. Collected data included patient's demographics, laterality, family history, age at diagnosis, presenting signs, treatment modalities, tumor stage, eye salvage rate, metastasis, and mortality. Results. Out of 200 retinoblastoma patients in our registry, $44(22 \%)$ patients were familial, 18 were probands, and 26 were second, third, or fourth affected family members. There were 76 affected eyes: 31 eyes of probands and 45 eyes of the other affected family members. Among probands, all patients $(100 \%)$ had at least one eye enucleated: $58 \%$ (18 eyes) of the affected eyes were enucleated and $32 \%$ (10 eyes) of the affected eyes were radiated. On the other hand, among the nonprobands, only $20 \%$ had one eye enucleated, and only 4 eyes (9\%) received radiation. The eye salvage rate was significantly higher in the nonprobands than in the probands in this series $(p=0.00206)$. Patients diagnosed by screening (38\%) had excellent visual outcome, and both eyes were salvaged. Conclusion. Awareness of families of the possibility of retinoblastoma and adequate screening led to a significantly higher rate of eye salvage in patients with familial retinoblastoma.

\section{Introduction}

Retinoblastoma $(\mathrm{Rb})$ is the most common childhood intraocular tumor, and $90 \%$ of the cases are diagnosed before the age of 5 years worldwide $[1,2]$. Globally, the incidence is approximately 1 in 15,000 to 20,000 live births $[3,4]$. An earlier epidemiological study from Jordan has reported an incidence of 9.32 cases per million children among $0-5$ years of age [4]. Approximately $20 \%$ of cases reported from Jordan are familial whereas only $10 \%$ of cases are familial in western countries $[4,5]$. The ratio of male : female was $2.3: 1$, and the number of bilateral cases was $47.5 \%$, which is higher than the bilateral $\mathrm{Rb}$ cases reported from western countries [4]. This might be because of the large-sized families in Jordan. The 2012 population census of Jordan has reported an average family household size of 5.1, thus increasing the incidence of familial $\mathrm{Rb}$ [6].

Over the years, the prognosis of $\mathrm{Rb}$ has improved because of better treatment modalities and early diagnosis. One of the most important poor prognostic factors for the management of $\mathrm{Rb}$ is the delay in diagnosis $[7,8]$. As patients in the developing countries are usually diagnosed in late stages of the disease, because of the difficulty in getting adequate health care, they present with a more advanced stage and, therefore, have less survival rates. The patients in the developed countries and those who have better socioeconomic status generally present at an earlier stage and get adequate health care leading to a higher survival rate as compared to patients from developing countries $[7,8]$.

Due to the rarity of the disease, people are generally not aware of the possibility of eye cancer [3]. The diagnosis of $\mathrm{Rb}$ in the first child (proband) is often delayed, but once the family becomes aware of the risk of developing $\mathrm{Rb}$ in the other newborns (nonprobands), they may consider any abnormal sign more seriously and, even more, they may get the nonprobands screened for earlier diagnosis and better outcome. We assume that the parental awareness may help in early diagnosis and better outcome among the 
nonprobands. Soliman et al., while conducting a study in Egypt, had proposed a similar hypothesis and has shown that awareness increased among parents with a proband child as compared to those without proband and further improved the outcome [9].

We aimed to evaluate the characteristics and management outcomes for the familial $\mathrm{Rb}$ cases in Jordan and the impact of awareness of these families on the detection and outcome of $\mathrm{Rb}$ in nonprobands.

\section{Materials and Methods}

This was a retrospective case series of 76 eyes of 44 consecutive familial $\mathrm{Rb}$ patients who had been managed in a single tertiary cancer centre in Jordan (KHCC). Patients enrolled between March 2008 and March 2016 were analyzed. Data was collected from the medical records and Ret-Cam images taken along with. Inclusion eligibility criterion included clinical and/or pathological diagnosis of Rb with a family history of $\mathrm{Rb}$. Thus, $\mathrm{Rb}$ patients without a family history of Rb were excluded from this study. The first family member with a diagnosis of $\mathrm{Rb}$ was called a proband, and other affected family members were called nonprobands. Information regarding the following parameters was collected: patients' age at diagnosis, gender, laterality, affected site, Reese-Ellsworth (RE) group at diagnosis, International Classification of $\mathrm{Rb}$ stage at diagnosis (Table 1), presenting signs and symptoms, management, eye salvage, visual outcome, metastasis, any other malignancy, and mortality. Selection and data collection required access to patients' medical records, Ret-Cam images, and medical reports for patients who were diagnosed and/or managed initially outside our centre. This study was approved by the institutional review board.

2.1. Treatment Methods. We used a combination chemotherapy regimen of CVE (carboplatin, vincristine, and etoposide). Each CVE cycle was repeated every 4 weeks for a total of 6 to 8 cycles according to patient's condition and tumor status. Ocular oncology follow-up was provided with examination under anesthesia before each and every cycle of chemotherapy and every 4 weeks thereafter. Fundus photos were taken using a Ret-Cam II (Clarity Medical System, Pleasanton, CA, USA). Combination focal therapy was applied as needed as transpupillary thermotherapy (TTT) and triple freeze thaw cryotherapy (MIRA CR 4000). External beam radiation therapy was administered when needed in a consistent fashion by applying $45 \mathrm{~Gy}$ in 25 fractions.

2.2. Statistical Analysis. Descriptive analysis was carried out using mean, median, and range. Comparative analysis was carried out between probands and nonprobands, and $p$ value was measured using Fisher's exact test for analyzing the predictive power of each factor.

\section{Results}

Out of the 200 retinoblastoma patients (between March 2008 and March 2016) admitted in the hospital, 44 (22\%) patients had familial Rb, 18 were probands, and 26 were second, third, or fourth affected family member (nonprobands). There were
TABLE 1: International intraocular Rb classification [1].

Group A: very low risk

Small discrete tumors not threatening vision

(i) All tumors are $3 \mathrm{~mm}$ or smaller, confined to the retina

(ii) Located at least $3 \mathrm{~mm}$ from the foveola and $1.5 \mathrm{~mm}$ from the optic nerve

(iii) No vitreous or subretinal seeding

Group B: low risk

No vitreous or subretinal seeding

(i) Tumors of any size or location not in group A

(ii) No vitreous or subretinal seeding

(iii) Subretinal fluid no more than $5 \mathrm{~mm}$ from tumor base

\section{Group C: moderate risk}

Focal vitreous or subretinal seeding and discrete retinal tumors of any size and location

(i) Local, fine, and limited seeding

(ii) Discrete intraretinal tumors of any size and location

(iii) Up to one quadrant of subretinal fluid

\section{Group D: high risk}

Diffuse vitreous or subretinal seeding

(i) Diffuse intraocular disseminated disease

(ii) Extensive or "greasy" vitreous seeding

(iii) Subretinal seeding may be plaque-like

(iv) More than one quadrant retinal detachment

Group E: very high risk

Very high risk with one or more of the following:

(i) Irreversible neovascular glaucoma

(ii) Massive intraocular hemorrhage

(iii) Aseptic orbital cellulitis

(iv) Tumor anterior to anterior vitreous face

(v) Tumor touching the lens

(vi) Diffuse infiltrating $\mathrm{Rb}$

(vii) Phthisis or prephthisis

76 affected eyes: 31 eyes for probands and 45 eyes for the other affected family members.

3.1. Proband Group. Out of the 31 affected eyes, 20 (64\%) were right eyes. The mean age at diagnosis was 17 months. Out of the 18 patients, 13 (72\%) were males and 5 (28\%) were females. Thirteen (72\%) patients had bilateral $\mathrm{Rb}$, and 5 (27\%) had unilateral Rb. Leukocoria was the most common presenting sign in $16(89 \%)$ patients, followed by strabismus in $2(11 \%)$ cases. All the affected eyes had multifocal disease. At the time of diagnosis, 26 (84\%) eyes were classified into group D or E and 5 (16\%) eyes were placed into group A, $\mathrm{B}$, or $\mathrm{C}[1]$.

Treatment and outcome: systemic chemotherapy was used in the management of $12(67 \%)$ patients. Focal therapy (TTT or cryotherapy) was applied in 11 (35\%) eyes, and $10(32 \%)$ eyes received external beam radiotherapy. Fourteen $(45 \%)$ eyes in this group were salvaged by the last 
TABLE 2: Clinical features and outcome of eye with retinoblastoma in both proband and nonproband groups.

\begin{tabular}{|c|c|c|c|c|c|c|}
\hline & & \multicolumn{2}{|c|}{ Proband } & \multicolumn{2}{|c|}{ Nonproband } & \multirow[t]{2}{*}{$p$ value } \\
\hline \multirow{3}{*}{ Presenting symptom } & Leukocoria & 15 & $83 \%$ & 10 & $38 \%$ & \\
\hline & Squint & 2 & $11 \%$ & 6 & $23 \%$ & \multirow[t]{2}{*}{0.1} \\
\hline & Screening & 0 & $0 \%$ & 10 & $38 \%$ & \\
\hline \multirow{5}{*}{ IIRC group at diagnosis } & $\mathrm{A}$ & 0 & $0 \%$ & 12 & $27 \%$ & \multirow{5}{*}{0.0001} \\
\hline & B & 1 & $3 \%$ & 14 & $30 \%$ & \\
\hline & $\mathrm{C}$ & 4 & $13 \%$ & 7 & $16 \%$ & \\
\hline & $\mathrm{D}$ & 20 & $65 \%$ & 8 & $17 \%$ & \\
\hline & $\mathrm{E}$ & 6 & $19 \%$ & 4 & $9 \%$ & \\
\hline \multirow{2}{*}{ Eye salvage rate** } & Yes & 14 & $45 \%$ & 36 & $80 \%$ & \\
\hline & No & 17 & $55 \%$ & 9 & $20 \%$ & \\
\hline \multirow{2}{*}{ BCVA } & Less than 0.5 & 6 & $18 \%$ & 5 & $20 \%$ & \\
\hline & Better than 0.5 & 8 & $26 \%$ & 13 & $50 \%$ & \\
\hline
\end{tabular}

IIRC: International Intraocular Retinoblastoma Classification; BCVA: best-corrected visual acuity at the last date of follow-up per eye.

${ }^{* *}$ Eye salvage rate per eye.

date of follow-up while enucleation was mandatory for 17 (55\%) of the treated eyes. In this group, all patients (100\%) had enucleation of at least one eye. At the last date of follow-up, best-corrected visual acuity was less than 0.5 in 6 (18\%) eyes and better than 0.5 in $8(26 \%)$ eyes (vision assessment was not possible for uncooperative patients 17 (55\%) eyes) (Table 2). One patient had secondary malignancy (maxillary osteosarcoma), and no case had metastasis or died by the last date of follow-up.

3.2. Nonproband Group. Twenty-six patients (59\%) of total cases of familial $\mathrm{Rb}$ were nonprobands. There were 45 affected eyes; 24 (53\%) of them were in the right eye. There were $11(42 \%)$ males and $15(58 \%)$ females. The mean age at diagnosis was 8 months. Eighteen (73\%) patients had bilateral disease, and the rest had unilateral disease.

Leukocoria was the presenting sign among 10 (38\%) patients, followed by strabismus among $6(23 \%)$ cases. Ten (38\%) patients had been diagnosed by screening. Thirtynine eyes (87\%) had multifocal disease, and 6 eyes (13\%) had unifocal disease. Twenty-six eyes $(57 \%)$ had tumor in stage A or B, and 19 eyes were grouped C, D, or E at the time of diagnosis [1].

Treatment and outcome: systemic chemotherapy was used for the management of 20 patients $(77 \%)$, while 6 patients (23\%) did not receive systemic chemotherapy. Focal therapy (TTT or cryotherapy) was applied to 35 eyes $(78 \%)$ in this group, and $10(22 \%)$ eyes did not receive focal treatment. Four (8\%) patients received external beam radiation. Thirty-six $(80 \%)$ eyes were salvaged at the last date of follow-up while enucleation was mandatory for $9(20 \%)$ of the treated eyes. Out of the 33 eyes in groups A, B, and C, 32 (97\%) eyes were salvaged while out of the 12 eyes in groups $\mathrm{D}$ and $\mathrm{E}$, only 4 (33\%) eyes were salvaged [1].

At the last date of follow-up, visual acuity was less than 0.5 in $5(20 \%)$ patients and better than 0.5 in $13(50 \%)$ patients (vision assessment was not applicable for $8(30 \%)$ patients) (Table 1). There was no case of secondary malignancy, or metastasis, and no deaths occurred up to the last date of follow-up.

\section{Discussion}

The results of the study are in favor of our hypothesis. We had hypothesized that an early diagnosis can be achieved among familial cases (other than the first affected patient in each family), which may further lead to better outcome. It is assumed that the families who have one child (probands) with retinoblastoma will be more aware after the birth of second or subsequent children. The results show that $58 \%$ of the nonproband cases were diagnosed as group A or B [1] (early intraocular stage), whereas only $3 \%$ of the probands were in these 2 groups. On the other hand, $84 \%$ of the probands were diagnosed in stage $\mathrm{D}$ or $\mathrm{E}$ (advanced intraocular stage), while only $26 \%$ of the nonprobands were diagnosed in these 2 groups. The mean age of diagnosis in the nonproband group was 8 months as compared to 17 months in the proband group, and 10 cases were detected by screening in the nonproband group. The earlier diagnosis among the nonprobands can be attributed to the awareness among the parents and their initiative to undertake screening. A similar retrospective study by Soliman et al. found that at the time of probands, none of the parents without probands knew about screening for $\mathrm{Rb}$ among newborns, whereas all parents with probands knew about it at the time of the second child [8]. The results of our study and those by Soliman et al. show that the screening for $\mathrm{Rb}$ among at risk patients helps in earlier diagnosis of $\mathrm{Rb}$ in countries like Jordan and Egypt, respectively [9]. This increasing trend of screening and awareness was significantly less than the developed world a few years back. Chantada et al. while comparing the screening rates among developing countries and the USA found that the screening of $\mathrm{Rb}$ was significantly less in developing countries (Argentina, Brazil, Jordan, Turkey, and Venezuela) [10]. 
The difference in the rate of salvage and other outcome parameters shows the impact of awareness and screening. Among the probands, there was enucleation of at least one eye with an eye salvage rate of $54 \%$, while in nonprobands who were diagnosed earlier, the eye salvage rate was significantly higher at $80 \%$. Retinoblastoma management guidelines recommend that the early diagnosed tumors (group A and few of group B tumors) can be treated with focal therapy even without chemotherapy, while more advanced tumors need chemotherapy and sometimes radiation and/or enucleation [11]. Our results show that in the proband group, 67\% of patients received chemotherapy, while the rest did not undergo chemotherapy because of late diagnosis and thus had to undergo enucleation. In contrast to this, $77 \%$ of the nonproband cases received chemotherapy and 23\% were treated by focal therapy as most of them had presented during the early stage. The enucleation rate was lower in the nonproband group. Soliman et al. have also shown that early eye screening decreased the tumor burden $(p=0.03)$, lowered the treatment burden $(p=0.04)$, and had a higher rate of ocular salvage $(p=0.01)$ and better visual outcome $(p=0.01)$ [9].

Our results have shown a younger mean age (17 and 8 months in the proband and nonproband groups, resp.) at the time of diagnosis as compared to earlier reports, which have shown the mean age to be 24 months [12]. In our study, approximately $80 \%$ of the cases were diagnosed before the age of 3 or 4 years. The younger age might be because of the parental awareness. We had earlier reported a median age of 12 months from a retrospective analysis of 10 years from our hospital for the patients enrolled until the year 2013 [13].

The signs and symptoms of $\mathrm{Rb}$ depend on its size and location. Leukocoria (which is seen when the tumor is large in size) is the most frequent presenting sign of $\mathrm{Rb}$, found in approximately $50-60 \%$ of cases, followed by strabismus (25\%) and inflammatory signs (6-10\%) [14-17]. In our groups, $83 \%$ of probands presented with leukocoria, followed by strabismus (11\%); on the other hand, in the nonproband group as $38 \%$ were discovered by screening, there were no presenting symptoms, while 38\% presented with leukocoria and $23 \%$ presented with squint. This difference in presentation again highlights the significance of awareness among the parents.

Early diagnosis and therefore management of retinoblastoma by focal therapy alone in the early stages rather than chemotherapy and possible radiation in the late stages will have an important impact on both social and economic status of the family and the community since focal therapy is much cheaper. In addition to that, early diagnosis means better visual outcome, so the affected patient will not be dependent in the future by keeping their eyes and vision and, therefore, will serve themselves without being an overload on the economy of the country specifically in the poor and developing countries.

Our results demonstrate the importance of early diagnosis of $\mathrm{Rb}$, which is possible through education of the community and training of health professionals. They can thus help in early identification, diagnosis, and initiation of management to improve the outcome. Results show that screening of the at risk population can be a diagnostic tool. In our study, patients diagnosed at an earlier stage had better outcome because the families were more aware of the signs of $\mathrm{Rb}$ and received medical care at an early stage. The retrospective study design is a limitation of our study. However, the long duration of 8 years as the inclusion period is a major strength of our study.

In conclusion, family awareness of the possibility of retinoblastoma and adequate screening (even in the absence of genetic testing) are associated with earlier diagnosis and higher rates of eye salvage in patients with retinoblastoma.

\section{Conflicts of Interest}

The authors declare that they have no competing interests.

\section{References}

[1] M. A. Linn, "Intraocular retinoblastoma: the case for a new group classification," Ophthalmology Clinic of North America, vol. 18, no. 1, pp. 41-53, 2005.

[2] J. Yun, Y. Li, C.-T. Xu, and B.-R. Pan, "Epidemiology and Rb1 gene of retinoblastoma," International Journal of Ophthalmology, vol. 4, no. 1, pp. 103-109, 2011.

[3] -201C;International Incidence of Childhood Cancer, Vol. II," IARC Scientific Publications, no. 144, pp. 1-391, 1998.

[4] I. Jaradat, Y. A. Yousef, M. Mehyar et al., "Retinoblastoma in Jordan: an epidemiological study (2006-2010)," Hematology/ Oncology and Stem Cell Therapy, vol. 4, no. 3, pp. 126-131, 2011.

[5] D. R. Lohmann and B. L. Gallie, Retinoblastoma, GeneReviews ${ }^{\circledR}$ [Internet], University of Washington, Seattle, Seattle (WA), 1993-2017, 2000 Jul 18 [updated 2015 Nov 19].

[6] Jordan Population and Family Health Survey, https:// dhsprogram.com/pubs/pdf/FR282/FR282.pdf.

[7] S. Canturk, I. Qaddoumi, V. Khetan et al., "Survival of retinoblastoma in less-developed countries impact of socioeconomic and health-related indicators," The British Journal of Ophthalmology, vol. 94, no. 11, pp. 1432-1436, 2010.

[8] K. E. Rodrigues, R. Latorre Mdo, and B. de Camargo, "Delayed diagnosis in retinoblastoma," Jornal de Pediatria, vol. 80, no. 6, pp. 511-516, 2004.

[9] S. E. Soliman, M. ElManhaly, and H. Dimaras, "Knowledge of genetics in familial retinoblastoma," Ophthalmic Genetics, vol. 18, pp. 1-7, 2016.

[10] G. L. Chantada, I. J. Dunkel, I. Qaddoumi et al., "Familial retinoblastoma in developing countries," Pediatric Blood \& Cancer, vol. 53, no. 3, pp. 338-342, 2009.

[11] Canadian Retinoblastoma Society, "National Retinoblastoma Strategy Canadian Guidelines for Care: Stratégie thérapeutique du rétinoblastome guide clinique canadien," Canadian Journal of Ophthalmology, vol. 44, Supplement 2, pp. S1-88, 2009.

[12] R. L. Hurwitz, C. L. Shields, J. A. Shields, P. Chévez-Barrios, M. Y. Hurwitz, and M. M. Chintagumpala, "Retinoblastoma," in Principles and Practice of Pediatric Oncology, P. A. Pizzo and D. G. Poplack, Eds., pp. 825-846, Lippincont-Raven, Philadelphia, 2003.

[13] I. Al-Nawaiseh, H. M. Jammal, Y. S. Khader, I. Jaradat, and R. Barham, "Retinoblastoma in Jordan, 2003-2013: ocular survival and associated factors," Ophthalmic Epidemiology, vol. 21, no. 6, pp. 406-411, 2014. 
[14] D. H. Abramson, C. M. Frank, M. Susman, M. P. Whalen, I. J. Dunkel, and N. W. Boyd, "Presenting signs of retinoblastoma," The Journal of Pediatrics, vol. 132, no. 3, pp. 505-508, 1998.

[15] A. Tarkkanen and E. Tuovinen, "Retinoblastoma in Finland 1912-1964," Acta Ophthalmologica, vol. 49, no. 2, pp. 293300, 1971.

[16] M. A. Bedfod, C. Bedotto, and P. A. Macfaul, "Retinoblastoma. A study of 139 cases," The British Journal of Ophthalmology, vol. 55, no. 1, pp. 19-27, 1971.

[17] A. Balmer and C. Gailloud, "Retinoblastoma: diagnosis and treatment. Including a clinical study," in Turning Points in Cataract Formation, Syndromes, and Retinoblastoma, W. Straub, Ed., pp. 36-96, Karger, New York, 1983. 


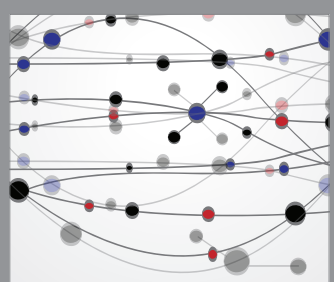

The Scientific World Journal
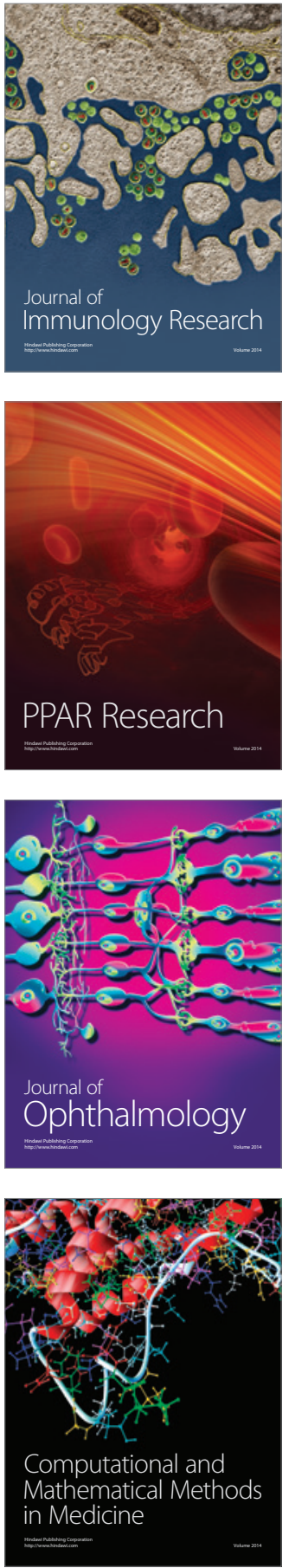

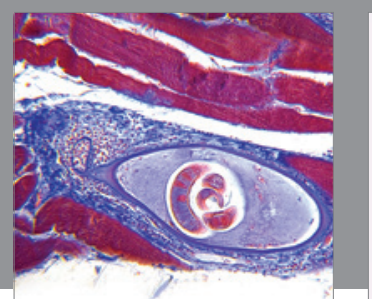

Gastroenterology Research and Practice
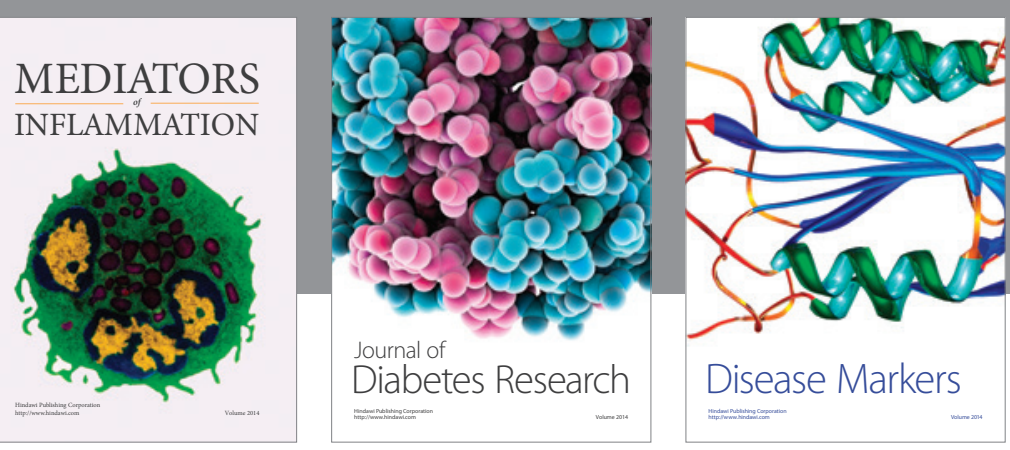

Disease Markers

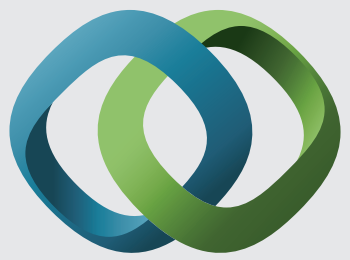

\section{Hindawi}

Submit your manuscripts at

https://www.hindawi.com
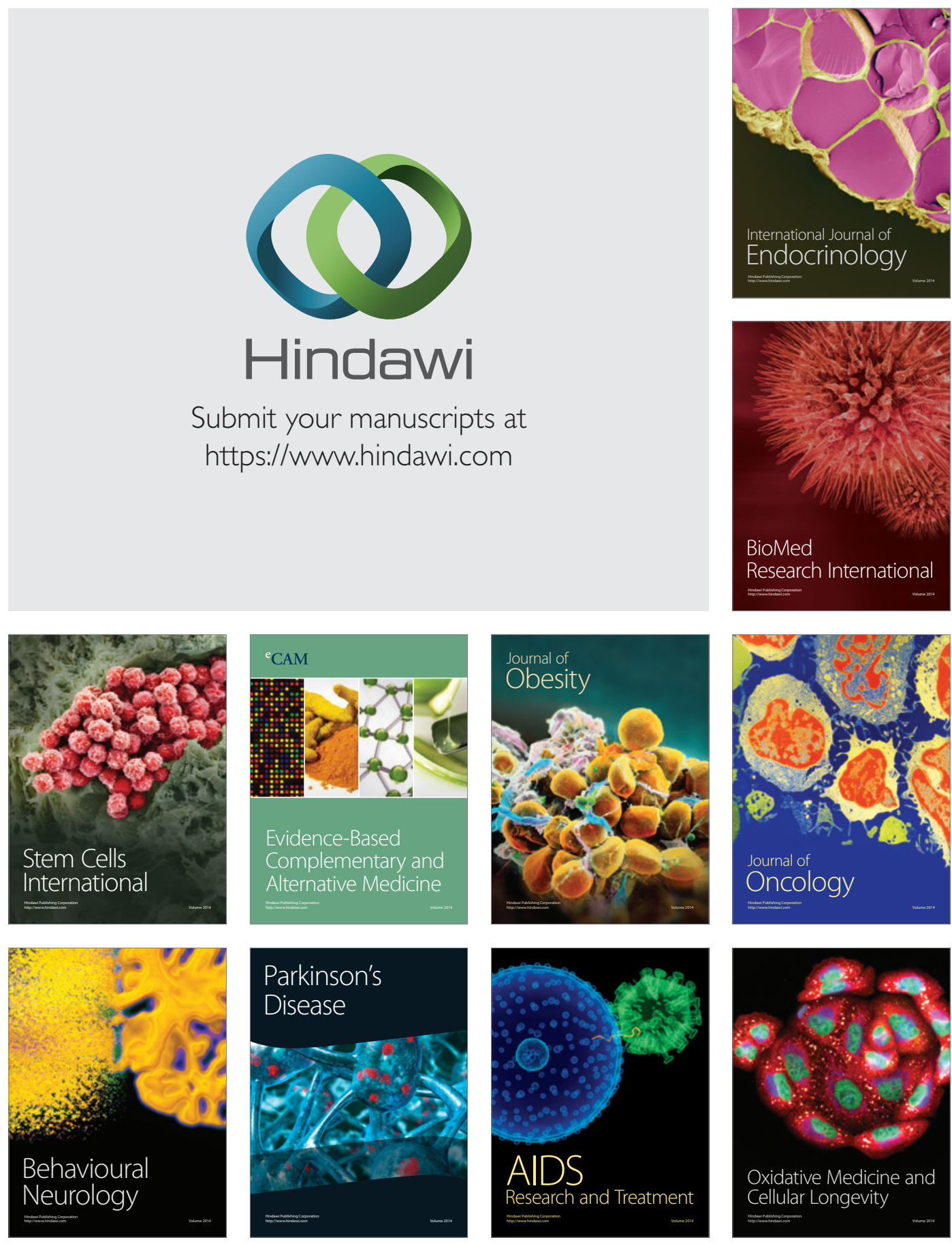